\title{
Design of Energy Efficient Wireless Sensor Networks through Overhead Reduction
}

\author{
B. Vengatesan \\ PG Student \\ Sri Venkateswara College of Engineering, \\ Sriperumbudur.
}

\author{
N. Kumaratharan \\ Professor \\ Sri Venkateswara College of Engineering, \\ Sriperumbudur.
}

\begin{abstract}
Wireless Sensor Networks (WSNs) are applied to different types of applications because of flexible and easy installation of sensor nodes. However, due to limited energy in the sensor nodes, so it is suitable for an application with low throughput requirement unlike cellular networks and mobile ad hoc networks. WSNs are battery dependency, there have been many methods used for efficiently utilizing battery of the sensor nodes, therefore increasing network lifetime. In existing they used joint contention and sleep control method. It can be increased network lifetime, but if increase the number of nodes the overhead to obtain the information is increased. The proposed algorithm can reduce overhead problem as well as improve network lifetime.
\end{abstract}

\section{Keywords}

Energy Adaptive Clustering Hierarchy (LEACH), Energy Efficient QoS Based Multipath routing protocol (EQSR), WSNs.

\section{INTRODUCTION}

Wireless Sensor networks (WSNs) are distributed and self configuring network consists of several sensor nodes for monitoring the physical and environment conditions. Sensor Node consists of sensing, computing, communication, actuation and power components. Sensor networks have recently emerged as an important computing platform. Sensor nodes are typically less mobile and more densely then mobile ad-hoc networks. Sensor nodes must be left unattended e.g. Hostile environment, which makes it difficult or impossible to recharge or replace their batteries. Network lifetime can be defined as the time elapsed until the first node or the last node in the network depletes its energy (dies).

Energy consumption in the sensor nodes can be due to either "useful" or "wasteful" sources. Useful energy consumption can be due to transmitting, sensing, receiving, and processing. Wasteful energy consumption can due to idle listening, retransmitting due to packet collision, overhearing and overhead. In our aim is saved the energy which is wasted in the wireless sensor networks. WSNs are used many application such as military surveillance and tracking, Environment monitoring and forecasting, healthcare etc.

Energy consumption and Energy balancing are one of the major issues for WSNs, because each node has limited energy and non-rechargeable. Many algorithms are used to improved energy consumption. They proposed the combination of clustering techniques (LEACH) and Energy Efficient QoS Based Multipath routing protocol (EQSR) are used for reducing overhead and energy consumption. Low Energy Adaptive Clustering Hierarchy (LEACH) protocol [1] is hierarchical routing based on clustering and find the optimal number of clusters in WSNs in order to reduced communication overheads, decreasing the overall energy consumption, interferences among sensor nodes and prolong the network lifetime. EQSR multipath routing protocol [4] uses the redidual energy, free buffer size and signal to noise ratio can predict the next hop through the paths construction phase. It splits up the transmitted message into a number of packets of equal size and adds correction codes and then transmits it over available multiple paths simultaneously to increase throughput, reduce end to end delay and information overhead.

\section{RELATED WORK}

LEACH is one of the energy efficient clustering algorithms $[5,6]$ for WSNs that forms node cluster based on the received signal strength and uses the local cluster heads transmits the data to cluster head or sink. Since data transfer to the sink consumes more energy, all the sensor nodes within a cluster take turns with the transmission by rotating the cluster heads. This leads to balanced energy consumption of all nodes and hence a longer lifetime of the networks.

Label Based Multipath Routing Protocol (LMR) [9] is proposed to reduce overhead, delay and energy consumption in WSNs. Wireless sensor networks typically consists of large number of nodes and work at very low data rate. Therefore, assigning globally unique IDs may be extremely expensive in terms of bandwidth and power consumption. Additionally, it is not necessary because these networks are data centric routing and specific node is not required. Similar to disjoint multipath and braided multipath LMR is designed to use only the localized information to find disjoint paths or segments to protect the working path. With one flooding, LMR can either find disjoint alternative paths to protect the working path. The flooding overhead is reduced by this LMR routing protocol. LMR can work with different data centric routing protocol.

\section{PROPOSED ALGORITHMS}

\subsection{Low Energy Adaptive Clustering Hierarchy}

LEACH is a cluster based protocol that applies randomized rotation of the cluster [5] head to distribute the energy load evenly among the sensor nodes in their cluster. The operation of LEACH is organized in rounds, each rounds consists of two phases that are i) setup phase ii) steady state phase.

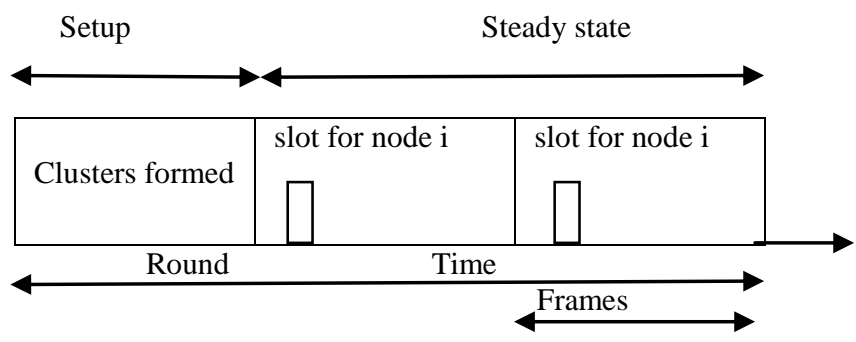

Figure 1. LEACH Operations. 


\subsubsection{Setup Phase}

In setup phase, the networks are divided into clusters, each with a randomly selected cluster head from nodes in a cluster. Each sensor node chooses a random number $m$ between 0 and 1. If $\mathrm{m}<\mathrm{T}(\mathrm{n})$ for node $\mathrm{n}$, the node becomes a cluster head. This decision is based on the threshold T (n).

$$
T(n)= \begin{cases}\frac{P}{1-P\left[r^{*} \bmod (1 / P)\right]} & \text { if } n \in G \\ 0 & \text { otherwise, }\end{cases}
$$

Where, $\mathrm{P}$ as the desired percentage of cluster heads $\mathrm{r}$ as the number of current round, $\mathrm{G}$ as the set of nodes that have not been cluster heads in last $1 / p$ rounds. In this protocol ensures that every nodes become a cluster head exactly once with in $1 / \mathrm{P}$ rounds.

\subsubsection{Steady State Phase}

During the steady state phase, the cluster head collects data [6] from nodes within their clusters respectively and aggregate the data before forwarding them directly to the sink or $\mathrm{CH}$. This is the formula of the amount of energy depletion by data transfer: the energy dissipated the transmitter $(\mathrm{E}$ elect $=$ $50 \mathrm{nj} / \mathrm{bit}$ ), the energy dissipation of the transmission amplifier $\left(\epsilon_{\text {amp }}=100 \mathrm{pj} / \mathrm{bit}\right)$.

Transmission cost $\mathrm{E}_{\mathrm{tx}}(\mathrm{k}, \mathrm{d})=\mathrm{E}_{\text {elect }} \mathrm{k}+\epsilon_{\mathrm{amp} \mathrm{kd}}{ }^{\kappa}$.

Receiving cost $\mathrm{E}_{\mathrm{rx}}(\mathrm{k})=\mathrm{E}_{\text {elect }}(\mathrm{k})$.

Where, $\mathrm{k}$ is the length of the message bits, $\mathrm{d}$ is the distance between nodes, $K$ represents path loss exponent $(K \leq 2)$.

\subsection{Energy Efficient QOS Based Multipath Routing Protocol}

EQSR routing in sensor networks will ensure guaranteed bandwidth or delay through the duration of connection as well as providing the use of most energy efficient path. To the best or our knowledge, no previous research has been addressed QoS routing in sensor networks. The proposed protocol extends the routing approach and considers only end to end delay. The protocol looks for a delay constrained path with the least possible cost based on a cost function defined for each link. Alternative paths with bigger costs are tried until one, which meets the end to end delay requirement and maximizes the throughput for best effort traffic is found. The protocol does not bring any extra overhead to the sensors. The EQSR routing protocol [4] performs routes discovery using multiple criteria such as energy remaining, remaining buffer size, signal to noise ratio. The proposed routing protocol designed especially for WSN, it can provided the service differentiation by giving real time traffic absolute preferential treatment over the non real time traffic. The proposed protocol uses the multipath paradigm together with a Forward Error Correction (FEC) technique to retrieve from node failures [2, 3] without invoking network wide flooding for path discovery. Since the flooding consumed more energy and consequently reduces the network lifetime.Eqsr protocol uses the residual energy, node free buffer size, signal to noise ratio to predict the next hop through the paths construction phase. It can spitted the transmitted message into a number of packets of equal size, adds forward correction codes, and then transmits it over multiple paths concurrently to raise the probability that an important portion of the packet is received at the destination without acquiring additional delay.

\subsubsection{Assumption}

Consider $\mathrm{N}$ identical sensor nodes are distributed randomly in the sensing area. All nodes have same transmission range and have enough battery power to carry their sensing, processing, communication activities. The sensor networks are fully connected and dens, therefore data can be sent from one node to another node in a multi-hop bases. Each node is assigned a unique ID and all nodes are willing to participate in communication process by forwarding data in the network. They assume that each node is able to compute it residual energy, its available buffer size, as well as record the link performance between itself and its neighboring node in terms of signal to noise ratio. By examine recent link performance of the data, predications and decisions about path stability may be made. They are going to find out the cost of every link, have to discover the path and finally select the path through which have send the messages.

\subsubsection{Link Cost Function}

The link cost function is used by the node to select the next hop during the path discovery phase and to use a cost function such as presented in with some changes. Let $\mathrm{Nx}$ is the set of neighbors of node $\mathrm{x}$. therefore in our cost function include an energy factor, available buffer size, interference factor with appropriate weights $(\alpha, \beta, \gamma)$.

Next hop $=\max _{\mathrm{y} \in \mathrm{Nx}}\left\{\alpha\right.$ Eresd $\left._{, \mathrm{z}}+\beta \mathrm{B}_{\text {buffer, } y}+\gamma \mathrm{I}_{\text {interference, } x y}\right\}$

Where, $E_{\text {resd, } y}$ is the current residual energy of node $y, B_{\text {buffer, }}$ is the available buffer size of node $y, \mathrm{I}_{\text {interference, } x y}$ is the signal to noise ratio for link between $\mathrm{x}$ and $\mathrm{y}$. in this cost function only consider the residual energy of node $\mathrm{y}$ but not $\mathrm{x}$. because node y consumes energy for data reception and transmission if it is selected as a next hop for node $\mathrm{x}$. they do not consider node $\mathrm{x}$, because node $\mathrm{y}$, node $\mathrm{x}$ still needs to spend the same amount of energy on data transmission. The total cost $\left(\mathrm{C}_{\text {total }}\right)$ for a path $\mathrm{P}$ consists of a set of $\mathrm{K}$ nodes in the sum of the individual link cost $\left(1_{(\mathrm{xy}) \mathrm{i}}\right)$ long the path.

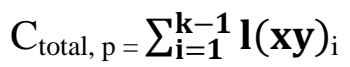

\subsubsection{Path discovery Phase}

The proposed protocol employs the queuing model presented in to handle both real and non real time traffic. Two different queues are used one is priority queue for real time traffic and other queue follows the first in first out (FIFO) basics for non real time traffic. Depend on the packet type, the classifier directs packet into the appropriate queue. The traffic allocation scheme first splits up the packets into a number of equal sized sub packets and then schedules sub packets simultaneously for transmission across the available multiple paths. Before scheduling the sub packets, the traffic allocation scheme adds forward error correction codes to improve the reliability of transmission and to increase the resiliency to paths failures and ensure that an essential portion of the packet is received by destination without any delay and more energy consumption through data retransmission at the sink, the parts are collected and reassembled, and get original message is recovered. The path discovery procedure [4] is executed according to the following phases:

\subsubsection{Initialization Phase}

Each sensor node broadcast a HELLO message through the network in order to have enough information about which of its neighbors can provided it with the highest quality data. Each node maintains and updates its neighboring table during this phase. The neighboring table contains information about the list of neighboring nodes of the sensor node. 


\begin{tabular}{|l|l|l|l|l|}
\hline $\begin{array}{l}\text { Source } \\
\text { Id }\end{array}$ & $\begin{array}{l}\text { Hop } \\
\text { count }\end{array}$ & $\begin{array}{l}\text { Residual } \\
\text { Energy }\end{array}$ & Free & $\begin{array}{l}\text { Link } \\
\text { Quality } \\
\text { Buffer }\end{array}$ \\
\hline
\end{tabular}

Figure 2: Initialization phase

\subsubsection{Primary Path Discovery Phase}

After initialization phase each sensor node has enough information to compute the cost function for its neighboring nodes. Then, the sink locally computes its preferred next hop node using the link cost function, and sends out a RREQ message to its most preferred next hop.

\begin{tabular}{|c|l|l|l|l|l|l|}
\hline src & Dest & Route & Residual & Free & Link & Route \\
id & Id & Id & Energy & Buffer & & Id \\
\hline
\end{tabular}

Figure 3: Primary path discovery phase

\subsubsection{Alternative Primary Path Discovery Phase}
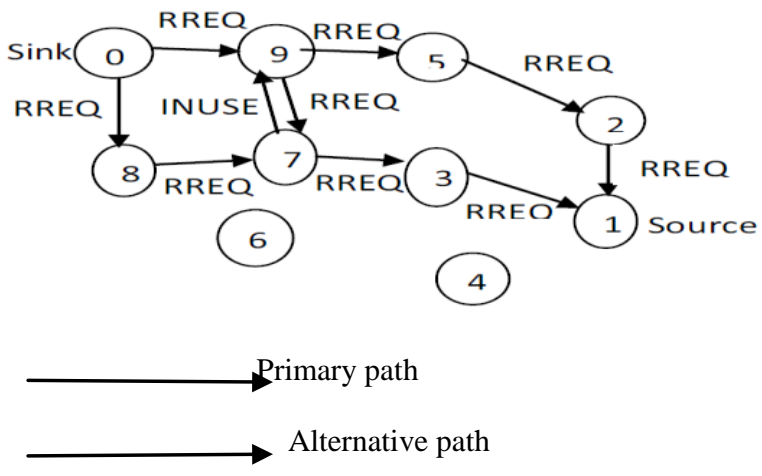

Figure 4: Alternative path discovery

For the second alternative path, the sink send alternate path RREQ message to its next most preferred neighbor. To avoid having paths with shared node, each node accepts only one RREQ message. For those nodes that receive more than one RREQ, only accept the first RREQ message.

\subsubsection{Paths selection}

After the completion of paths discovery phase and the paths have been constructed, they need to select a set of paths from the $\mathrm{N}$ available paths to transmit the packets from the source or cluster head $(\mathrm{CH})$ to destination with desired bound of data delivery given by $\alpha$. To find the number of required paths, they assume that each path is associated with some rate $p_{i}$ $(\mathrm{i}=1,2, \ldots \mathrm{N})$ that corresponds to the probability of successfully delivering a message to the destination.

$$
k=x_{\alpha} \sqrt{\sum_{i=1}^{N} P_{i}}(1-P i)+\sum_{i=1}^{N} p i
$$

Here, $\mathrm{x}_{\alpha}$ is the corresponding bound from the standard normal distribution for different level of $\alpha$. Table 1 lists [10] some values for $\mathrm{x}_{\alpha}$
Table 1. Some Values For Different Bounds.

\begin{tabular}{|c|l|l|l|l|}
\hline $\mathrm{A}$ & $95 \%$ & $90 \%$ & $85 \%$ & $50 \%$ \\
\hline $\mathrm{X} \alpha$ & -1.65 & -1.25 & -1.03 & 0 \\
\hline
\end{tabular}

\section{SIMULATION RESULTS}

In this section, they evaluate the performance of our proposed algorithm through the simulations. In this results are compared our proposed (LEACH-EQSR) algorithm with LEACHAODV based on four performance metrics: total energy consumption, overhead, throughput and network lifetime. The reference network of our simulations consists of 100 nodes distributed randomly in an area of $50 \mathrm{~m} \times 50 \mathrm{~m}$. The simulation results and comparison of the proposed system were executed and analyzed using Network Simulator (ns-2) Figure 5 shows the performance comparisons between LEACH-AODV and LEACH-EQSR routing protocol in WSNs. The Overhead is increased the overall network lifetime is decreased. So the proposed protocol has performance better than existing protocol in network lifetime.

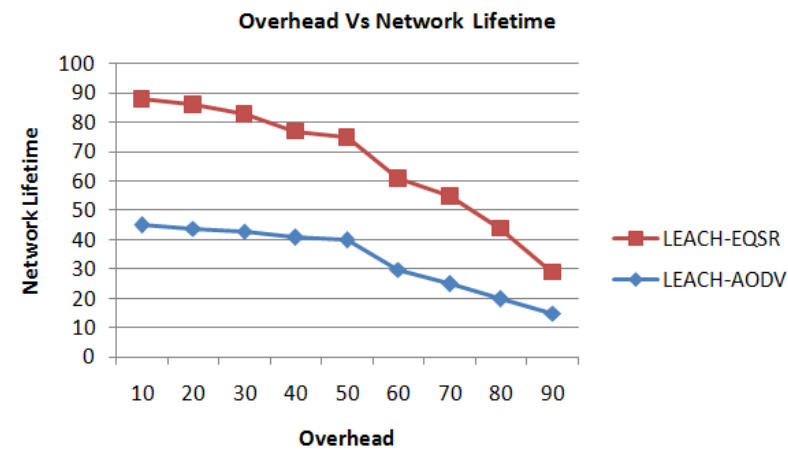

Figure 5: Network lifetime vs. Overhead

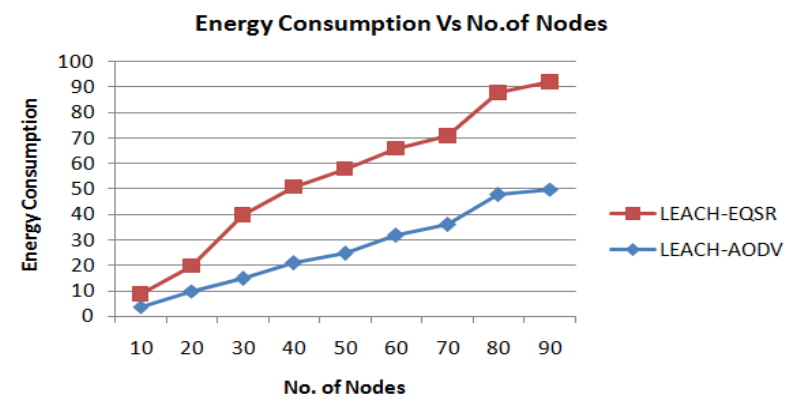

Figure 6: Energy Consumption vs. No. of Nodes

Figure 6 shows the results for energy consumption in LEACH-EQSR and LEACH-AODV. As it can be seen, the proposed protocol is enhanced energy consumption comparisons to the existing protocol. 


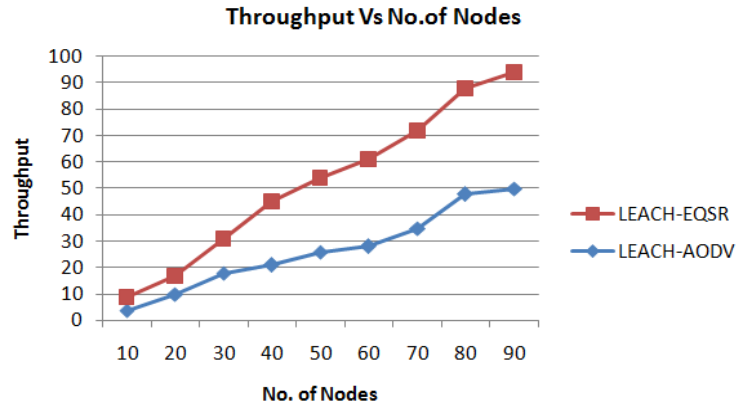

Figure 7: Throughput vs No.of nodes

Figure 7 shows the proposed(LEACH-EQSR) protocol is upgraded throughput compare then exiting algorithm. The number of nodes are increased, corresponding the throughput also increased.

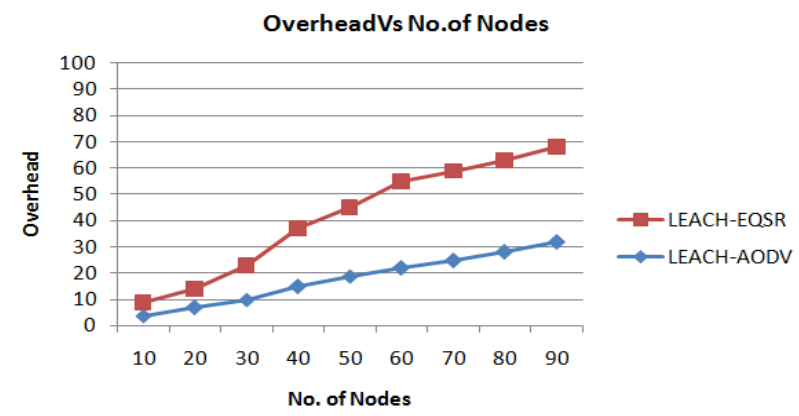

Figure 8: Overhead Vs No.of Nodes

Figure 8 shows the performance comparision between LEACH-AODV and LEACH-EQSR routing protocol in WSNs. The number of nodes are increased, the overhead also increased. The proposed algorithm is reduced overhead compare than the exiting algorithm.

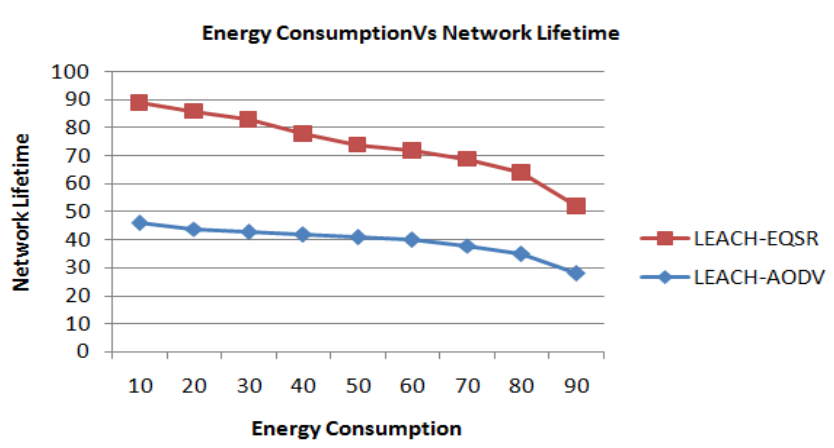

Figure 9: Energy consumption vs. Lifetime

The figure 9 shows, the energy consumption is increased, but the lifetime is decreased. The proposed algorithm is improved network lifetime compare than the exiting algorithm.

\section{CONCLUSION}

The combination of LEACH and EQSR Multipath routing protocol are proposed for wireless sensor networks for prolonging overall network lifetime by reducing overhead and energy consumption. In the proposed LEACH protocol is form the cluster and elect the cluster head, in the cluster head is forwarded the sub packets to the neighboring node. The EQSR routing protocol is used to split the transmitted message into number of sub packets of equal size adds correction code and then transmits it over multiple paths simultaneously. In this proposed algorithms can be improved network lifetime. The results are compared using Network Simulator (ns-2)

\section{REFERENCES}

[1] Heinzelman.W, A.Chandrakasan, and H.Balakrishnan, "Energy Efficient Communication Protocol for Wireless Sensor Networks", Proc. Hawaii Conference System Science, jan.2000.

[2] R.Devisri and R.J.Archchana Devy, "Reliable and Power Relaxation Multipath Routing Protocol for Wireless sensor Networks", International Conference on Advancements in Information Technology, Vol.20, 2011.

[3] Ying Hong Wong, Chih Hsiao Tsai, Hue Min Huang, "A Hierarchy Based Multipath Routing Protocol For Wireless Sensor Networks", Information and Management Sciences, Volume.19, Number.2, pp.353366, 2008.

[4] Ram Kumar E and Vinothraj N , Kiruthiga G "Energy Based Multipath Routing Protocols for Wireless Sensor Networks" Journal of Computer Applications ISSN: 0974 - 1925, Volume-5, Issue EICA 2012-2, February $10,2012$.

[5] Erfan.Arbab, Vahe.Aghazarian, Alireza.Hedayati, and Nima.Ghazanfari Motlagh, "A LEACH Based Clustering Algorithm for Optimizing Energy consumption in Wireless Sensor Networks", International conference on Computer Science and Information Technology, April2012.

[6] M.J.Handy, M.Haase, and D.Timmermann, "Low Energy Clustering Hierarchy With Deterministic Cluster Head Selection", International Workshop on Mobile and Wireless Communication Network, pp.368-372, 2002.

[7] Sridhar Manda, Archana $\mathrm{N}$ and Umarani $\mathrm{N}$, "Maximizing the Lifetime of Wireless Sensor networks Using CRT Based Packet Splitting Algorithm", International Journal of Advancements in Research and Technology, Volume.2, April-2013.

[8] I.F.Akyildiz, W.Su, Y.Sankarasubramanian, and E.Cayirci, "Wireless Sensor Networks: A Survey", Computer Networks, Vol.38, no.4,pp. 393-422, 2002.

[9] Xiaobing Hou, David Tipper, and Joseph Kabara, "Label Based Multipath Routing in Wireless Sensor Networks", IEEE Trans. Mobile Comput., vol.9, pp.1508-1521, 2008.

[10] Ali Ranjide Rezaie and Mirkamal Mirnia "CMQ: Clustering based multipath routing algorithm to improving QoS in wireless sensor networks", International journal of computer science, Vol. 9, Issue 3, No.1, May 2012. 